\title{
Research Paper \\ The Psychometric Properties of Body Shape Questionnaire 8C in Women With Eating Disorders
}

\section{*Fateme Veisy ${ }^{1}$, Seyed Mojtaba Ahmadi², Kheirollah Sadeghi ${ }^{3}$, Mansour Rezaee}

1. PhD Student in Clinical Psychology, School of Behavioral Sciences and Mental Health (Tehran Institute of Psychiatry), Iran University of Medical Sciences, Tehran, Iran 2. PhD Student of Clinical Psychology, Department of Clinical Psychology, Student Research Committee, Shahid Beheshti University of Medical Sciences, Tehran, Iran 3. PhD in Clinical Psychology, Assistant Professor, Department of Clinical Psychology, Faculty of Medicine, Kermanshah University of Medical Sciences, Kermanshah, Iran. 4. PhD in Biostatistics, Associate Professor, Department of Biostatistics, Faculty of Public Health, Kermanshah University of Medical Sciences, Kermanshah, Iran.

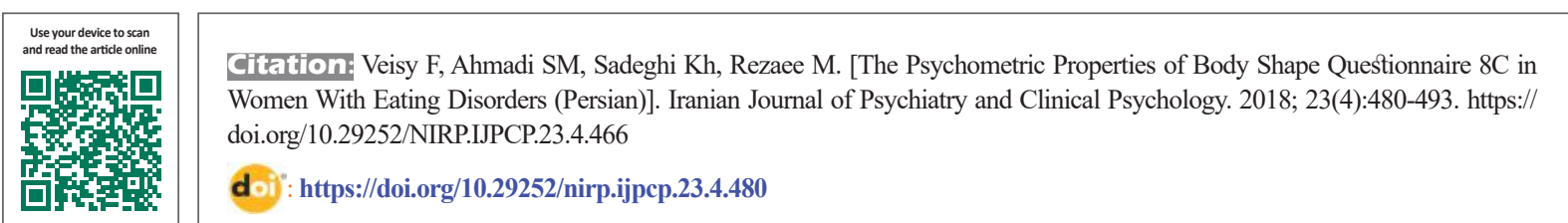

Received: 14 Nov. 2015

Accepted: 25 Feb. 2017

Key words:

Body image, Body

Shape Question-

naire 8C, Eating

disorders, Validity \&

Reliability, Cut point

\section{A B S T R A C T}

Objectives Extreme concern about body shape is the main feature of eating disorders. Eating disorders co-occur with several medical and mental disorders. So the use of a valid and reliable tool for measuring and preventing this disorder and its consequence seems necessary (or very helpful). Therefore, the aim of the present study was to examine psychometric properties of the Body Shape Questionnaire 8C (BSQ$8 \mathrm{C}$ ) in a sample of women with eating disorders.

Methods The main tool of this cross-validation study, i.e. BSQ-8C, was translated to Persian. Then psychometric properties of the scale were studied in a sample of women with eating disorders who were selected by convenient sampling method. Data collecting tools were BSQ-8C, BSQ-34, Body Dysmorphic Meta Cognition Questionnaire (BDMCQ), and Yale-Brown Obsessive Compulsive Scale for Body Dysmorphic Disorder (YBOCS-BDD). For data analysis with SPSS version 16, correlation coefficient, Cronbach's alpha, split half coefficient, and ROC curve were used.

Results In the sample of women with eating disorders the coefficients of Cronbach's alpha and split-half were 0.79 and 0.74 , respectively. Concurrent validity by way of correlation between the scale and BSQ34, YBOCS-BDD, and BDMCQ were 0.91, 0.59 and 0.58, respectively. Sensitivity, specificity, and the cut point of this questionnaire were $0.80,0.67$ and 26.5 , respectively.

\section{Extended Abstract}

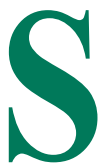

\section{Introduction}

childer (1935) for the first time discussed the concept of body image from a psychological point of view. Body image is defined as the mental image of our body. The multidimensional concept of body image includes understanding, perception, and attitude toward body [1-2]. The body shape concerns are common among adolescents, especially in western cul- ture. In its more severe forms, it is among the main causes of eating disorder, Anorexia Nervosa (AN), and Bulimia Nervosa (BN). It has been emphasized in DSM [3].

The complicated concept of body image disturbance involves two relevant and yet distinct concepts: body size overestimation and body shape concern [4]. In 1987, Cooper et al. designed the first tool to measure body shape concerns and termed it as the Body Shape Questionnaire (BSQ). Its differential validity, interna consistency, and test-retest reliability were confirmed

* Corresponding Author:

Fateme Veisy, PhD Student

Address: Department of Clinical Psychology, School of Behavioral Sciences and Mental Health (Tehran Institute of Psychiatry), Iran University of Medical Sciences, Tehran, Iran.

Tel: + 98 (917) 3294610

E-mail: veisy.fateme@gmail.com 
in this study designed for the North American population [3]. A great advantage of this questionnaire is raising questions to measure the major syndromes of body image [6]. This questionnaire has been widely used in the areas of clinical medicine [7], demography [8], intervention [9] and prevention [10]. The body shape questionnaire has also been widely used in populations with eating disorders $[11,12]$ and those without eating disorder, including female students from different racial and ethnic groups [13, 14], teenage boys and girls [15], people on diet [16], and older women [17].

The validity and reliability of this questionnaire have been confirmed by various studies [3, 5, 6, 18-21]; however, this questionnaire is very long, and it is rarely used in multistage studies or with other questionnaires [22]. Several short versions of the BSQ have been proposed; however, only the BSQ-8C version has been highly sensitive to change [23]. It is important to note that body shape dissatisfaction is among the main risk factors of eating disorders [24]. Eating disorders may be associated with mental disorders such as depression, anxiety, occupational and social dysfunction, and decreased quality of life [25]. Therefore, to measure body shape dissatisfaction in each culture, specific credible and reliable tools must be used. The psychometric properties of BSQ-8C have not been investigated in Iran; however, eating disorders are influenced by the cultural context of societies. Considering the application of this questionnaire in clinical and research areas, this study investigated the psychometric properties of the BSQ-8C in a sample of women with eating disorders.

\section{Method}

This cross-sectional and validation study was conducted in spring 2014. The BSQ-8C was the main tool used for collecting data. The questionnaire was translated from English to Farsi and then from Farsi to English, and necessary corrections were made in the Farsi version. Using convenience sampling, 63 individuals were selected from beauty and fitness centers of Kermanshah, Iran. Informed consent was obtained from the participants. Two clinical psychologists conducted independent diagnostic interviews, and according to the diagnostic criteria of DSM-IV-TR, 46 individuals were diagnosed with eating disorder. The participants were then asked to fill out demographic characteristics forms, BSQ-8C, BSQ-34, YBOCS-BDD, and BDMCQ.

\section{Body Shape Questionnaire-8C (BSQ-8C)}

The BSQ-8C is a short version of the body shape questionnaire. It also contains eight questions extracted from the full version of Evans and Dolan's questionnaire in 1993. The BSQ-8C version has been widely used for its high sensitivity to changes with time periods. Welch et al. (2012) conducted two studies to investigate the psychometric properties of the BSQ-8C. The results showed high values of test-retest reliability, internal consistency, and convergent validity [26].

\section{4-Item Body Shape Questionnaire (BSQ-34)}

The 34-item Body Shape Questionnaire (BSQ-34) questionnaire measures body weight, shape dissatisfaction, and its subsequent obsessive mental preoccupation and distress. Higher scores indicate higher levels of dissatisfaction. This questionnaire was designed in a clinical population, including patients with anorexia nervosa and bulimia nervosa, and women without eating disorders. Body shape questionnaire is a valid and reliable scale of body image with suitable test-retest reliability [3]. The concurrent validity of this questionnaire was confirmed through correlation with other body image scales such as the Body Dysmorphic Disorder Examination (BDDE) and the Multidimensional Body-Self Relations Questionnaire (MBSRQ) [6].

\section{The Body Dysmorphic Meta-Cognition Questionnaire (BDMCQ)}

The 31-item Body Dysmorphic Meta-Cognition Questionnaire (BDMCQ) consists of four factors: metacognitive control strategies (on appearance), objectification of thoughts (blending thoughts), positive and negative metacognitive beliefs, and safety behaviors on body deformity. Participants report their thoughts and perceptions about their body deformity over the past two weeks in the form of a 4-point Likert scale. Rabiei et al. (2011) confirmed the concurrent validity of this questionnaire. Suitable diagnostic validity and reliability were reported for this questionnaire [27].

The Yale-Brown Obsessive Compulsive Scale modified for Body Dysmorphic Disorder (YBOCS-BDD)

The 12-item Yale-Brown Obsessive Compulsive Scale modified for Body Dysmorphic Disorder (YBOCS-BDD) measures the intensity of the symptoms of body dysmorphic disorder. Philips al. (1997) conducted a study on 125 outpatients, and the results of the factor analysis revealed the significance of intellectual-practical obsession and two questions related to insights, including beliefs about appearance and avoidance evaluation [28]. In Iran, Rabiei et al. supported the concurrent reliability and validity of this questionnaire. The results of the factor analysis of this questionnaire revealed the significance of obsessive com- 
pulsion about appearance and the power of thought control [29]. Data were analyzed in SPSS 16, using frequency, mean, standard deviation, Cronbach's alpha coefficient, correlation coefficient, and Receiver Operating Characteristic (ROC) curve (Figure 1).

\section{Results}

Two clinical psychologists conducted independent diagnostic interviews with 63 individuals. Among them, 2 individuals were diagnosed with anorexia nervosa, 20 individuals were diagnosed with bulimia nervosa, 24 individuals were diagnosed with unknown eating disorders, and 17 others showed no symptom of eating disorders.

To measure the validity and reliability of the body shape questionnaire, 46 individuals with eating disorders were examined. Using Cronbach's alpha coefficient and splithalf method, the reliability of BSQ- $8 \mathrm{C}$ were found as 0.79 and 0.74 , respectively.

As seen in Table 1, a significant correlation found between the BSQ-8C and the total score of the BSQ-34 ( $<<0.001)$. There were also significant correlations between this questionnaire and the BDMCQ and the subscales of meta-cog- nitive control strategies, thought-action fusion and positive and negative metacognitive beliefs in women with an eating disorder $(\mathrm{P}<0.001$ and $\mathrm{P}=0.001)$. However, there was no significant correlation between the BSQ- $8 \mathrm{C}$ and the subscale of safety behaviors. In addition, there were significant correlations $(\mathrm{P}<0.001)$ between this questionnaire, the total score of the YBOCS-BDD, and the subscale of obsessive compulsion about appearance in women with eating disorders. However, no significant correlation existed between the BSQ- $8 \mathrm{C}$ and the subscale of power of thought control.

The cut-off point, sensitivity, specificity, efficacy, and the Overall Misclassification Rate (OMR) of the BSQ- $8 \mathrm{C}$ are shown in a sample of women with eating disorders in Table 2. As shown in the Table 2, the best cut-off point was found to be 26.5 .

\section{Discussion}

Our findings suggest the reliability of the BSQ-8C was suitable, using both Cronbach's alpha coefficient and splithalf method. Similarly, Welch et al. (2012) studied a group of students and confirmed the reliability and internal consistency of the BSQ-8C using test-retest method and Cronbach's alpha coefficient, respectively [26]. Pook et al. in

Table 1. Concurrent validity of the BSQ-8C in women with eating disorder

\begin{tabular}{|c|c|c|c|}
\hline & Scale & $r$ & $\mathbf{P}$ \\
\hline & BSQ-34 & 0.91 & $P<0.001$ \\
\hline \multirow{5}{*}{ BDMCQ } & Total score & 0.59 & $P<0.001$ \\
\hline & Meta-cognitive control strategies & 0.57 & $P<0.001$ \\
\hline & Thought-action fusion and & 0.54 & $P<0.001$ \\
\hline & Positive and negative metacognitive beliefs & 0.47 & 0.001 \\
\hline & Safety behaviors & 0.28 & 0.059 \\
\hline \multirow{3}{*}{ YBOCS-BDD } & Total score & 0.58 & $P<0.001$ \\
\hline & Obsessive compulsion about appearance & 0.65 & $P<0.001$ \\
\hline & Power of thought control & 0.12 & 0.425 \\
\hline
\end{tabular}

Table 2. The cut-off point, sensitivity, specificity, efficacy and the Overall Misclassification Rate (OMR) of the BSQ-8C

\begin{tabular}{cccccc}
\hline & & The Area & & & Overall \\
& Cut-Off Point & $\begin{array}{c}\text { Under the } \\
\text { Curve }\end{array}$ & Sensitivity & Specificity & $\begin{array}{c}\text { Efficacy } \\
\text { Misclassification } \\
\text { Rate (OMR) }\end{array}$ \\
\hline BSQ-8C & 26.5 & 0.76 & 0.80 & 0.67 & 0.71 \\
\hline
\end{tabular}




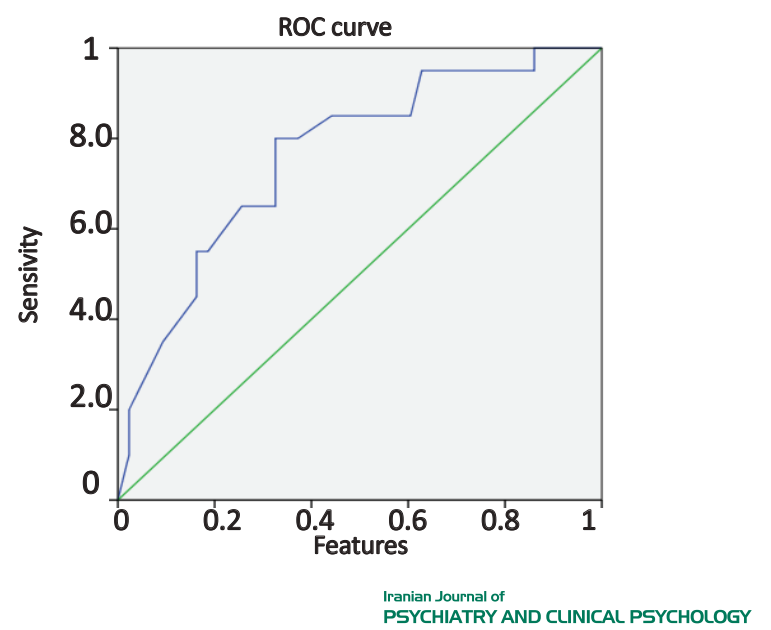

Figure 1. ROC curve of BSQ-8C

Diagonal segments are produced by ties

their study, confirmed the reliability of the BSQ-8C [23]. The correlation between the BSQ- $8 \mathrm{C}$ and BSQ-34 was very high, which indicates that the BSQ- $8 \mathrm{C}$ can replace the BSQ-34 to facilitate achieving research and clinical goals.

The correlation between the BSQ-8C and BDMCQ was very high in women with eating disorders. This correlation is justified as the metacognitive assessment involves any kind of knowledge or cognitive process associated with cognitive assessment or control [27]; therefore, the BDMCQ measures these cognitions in relation to the body shape [27]. On the other hand, serious concern about the shape of the body is a significant indicator of eating disorders [3] and people with eating disorders rely mainly on their body shape, weight, eating habits, their ability to control them, and evaluate themselves accordingly [25]. Therefore, the correlation obtained was reasonable, and it confirmed the suitable concurrent validity of the BSQ-8C.

There was a significant correlation between the BSQ-8C and YBOCS-BDD in women with eating disorders. The positive and significant correlation between these two questionnaires can be explained: selective attention is the first mechanism that maintains body image disturbance. Selective attention to some parts of the body increases obsessive mental preoccupation and creates a negative cognition, when encountering those parts of the body [25]. Therefore, this correlation appears reasonable and confirmed the suitable concurrent validity of the BSQ-8C in women with an eating disorder.

Previous study has also confirmed the validity of the BSQ$8 \mathrm{C}$. Welch et al. measured and confirmed the convergent validity of the BSQ-8C [26]. However, the cut-off point of the BSQ-8C has not been calculated in previous studies. The cut-off point was shown to be 26.5 in this study.
This study aimed to develop information on body and body shape dissatisfaction in a sample of women with eating disorders and confirmed the reliability and validity of the BSQ-8C. In the original and long version of the body shape questionnaire, some questions are not suitable for men; therefore, it is not an appropriate questionnaire for studying men [30]. On the other hand, the questions designed in BSQ-8C are appropriate for both sexes; hence, this questionnaire can be used to measure body shape dissatisfaction in healthy men with eating disorders. Pieces of evidence such as satisfactory psychometric properties of the BSQ-8C, high correlation between this questionnaire, and the original and long version, sensitivity to treatment, suitable questions, and brevity suggests that this questionnaire is an appropriate tool for assessing body shape dissatisfaction. Therefore, BSQ-8C is suggested to be used in nonclinical and clinical studies, such as treatment evaluation.

The present study examined a sample of women with eating disorders; therefore, the findings should be generalized to other populations with caution. Future studies should include other clinical populations as well as men. It is also recommended to conduct studies to measure other types of validity and reliability of the BSQ-8C, which were not used in this study.

\section{Ethical Considerations}

\section{Compliance With Ethical Guideline}

All participants completed the informed consent form.

\section{Funding}

This research was funded (No: 93064) by Kermanshah University of Medical Sciences.

\section{Conflict of Interest}

The authors declare no conflict of interest.

\section{Acknowledgments}

The authors hereby would like to express their appreciation to all the esteemed professors and all the participants for their sincere cooperation. 


\title{
مشخصات روانسنجى يرسش نامه شكل بدن AC در زنان مبتلا به اختلال خوردن
}

\author{
"فاطمه ويسى'، سيد مجتبى احمدى"، خيراله صادقى"، منصور رضايى"

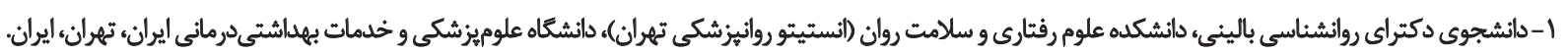

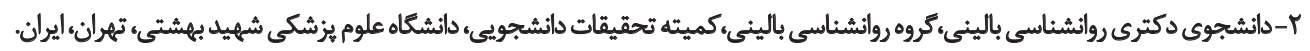

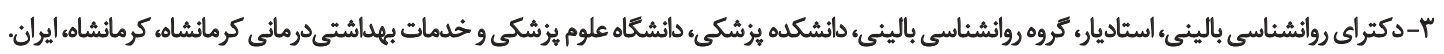

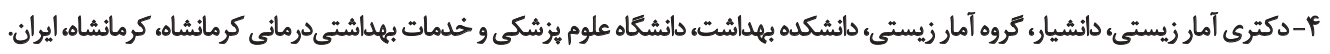

\begin{abstract}
حكטי

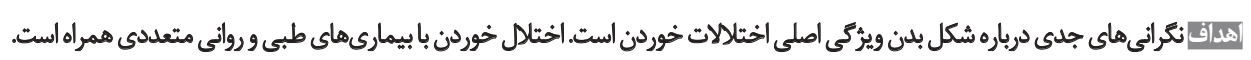

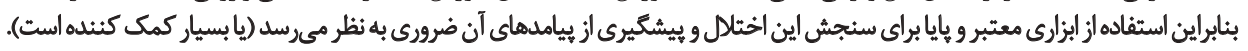

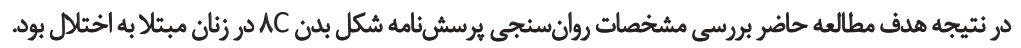

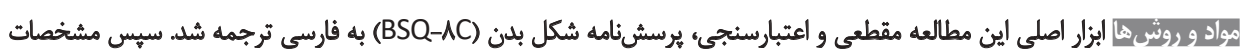

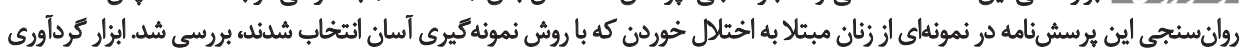

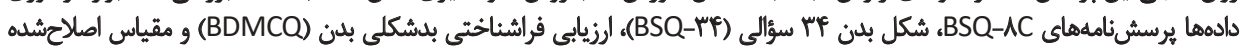

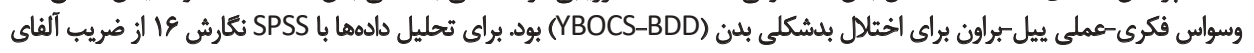

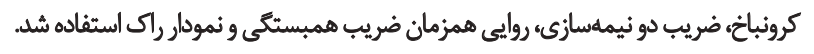

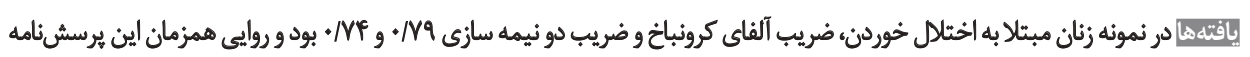

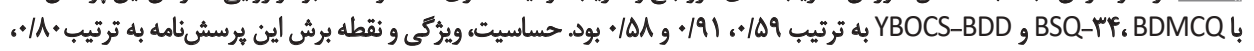

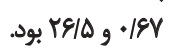

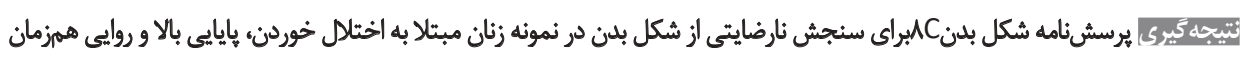

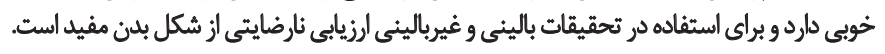

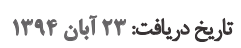
تاريخ بذيرش: V+ اسفيند هوبان

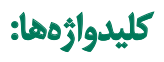
تصوير بدنء برنيشناميه

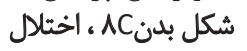

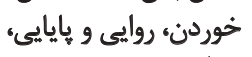
نقطمه برش رون

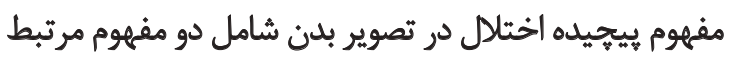

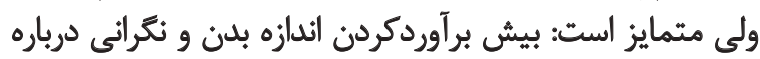

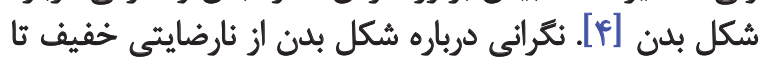

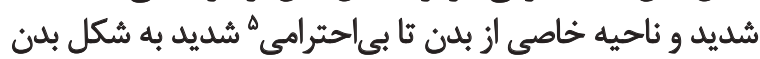

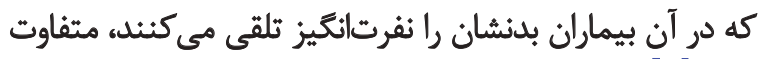

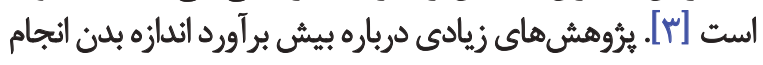

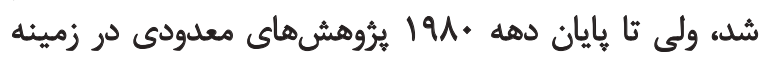

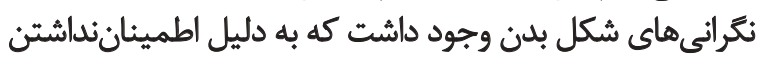

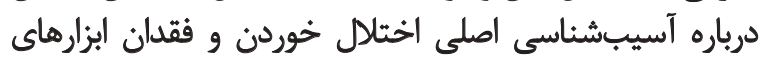

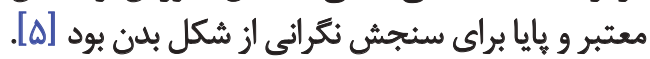

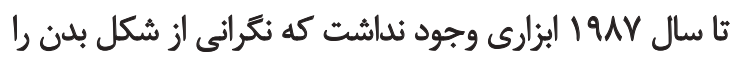

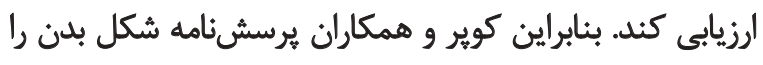

5. Disparagement dales

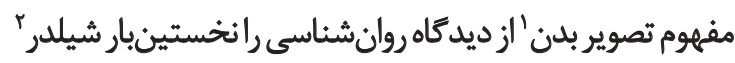

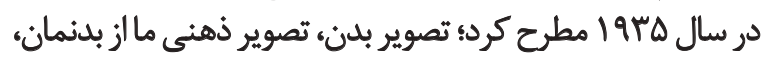

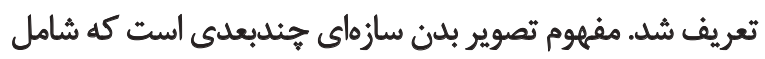

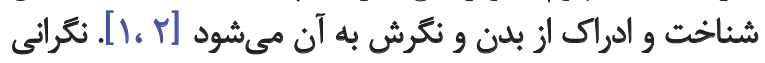

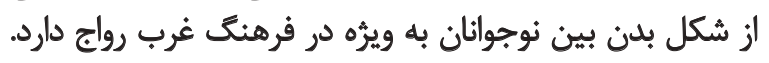

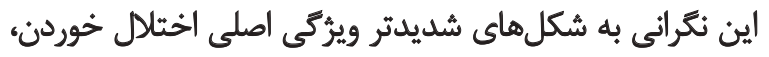

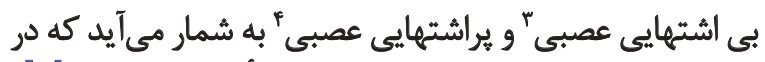

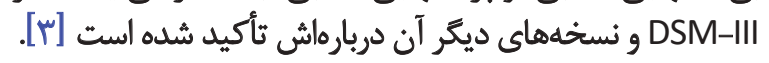

\footnotetext{
1. Body image

2. Schilder

3. Anorexia Nervosa (AN)

4. Bulimia Nervosa (BN)
}

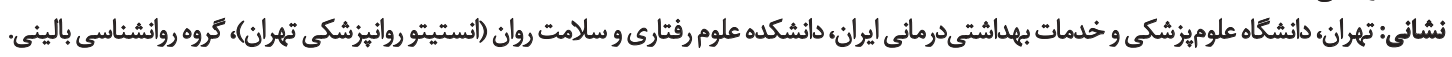

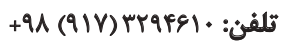
veisy.fateme@gmail.com يلكت الكترونيكي: 
روانسنجى يرسشنامه شكل بلن AC در ايران بررسى نشده

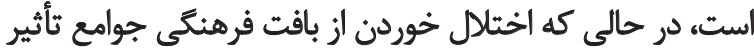

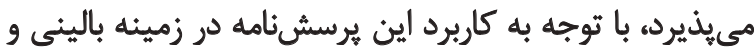

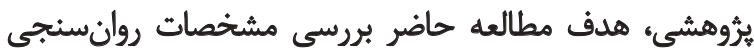
يرسشنامه شكل بدن AC در نمونهاى از زنان مبتلا بـ به اختلال

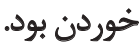

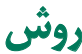

مطالعه حاضر از نوع مقطعى و اعتبارسنجى است كه در بهار

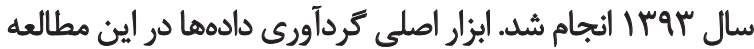

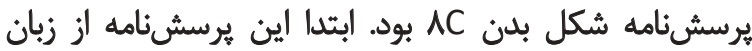

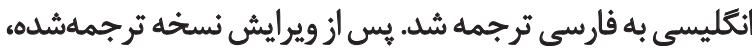

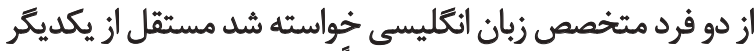

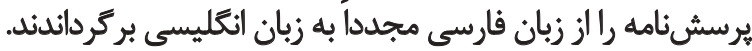

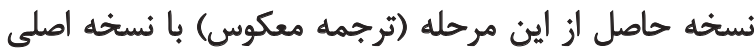

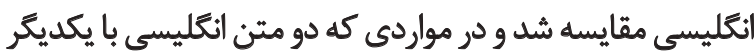

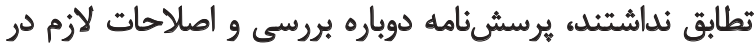
نسخه فارسى انجام شد.

زامعه آمارى يُوهش حاضر تمامى مراجعه كنيند

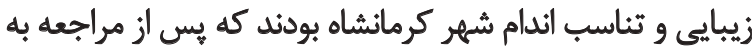

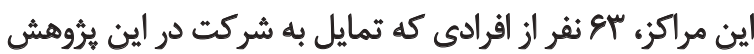

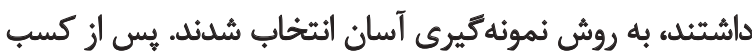

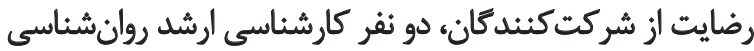

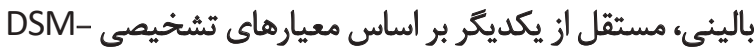

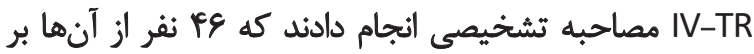

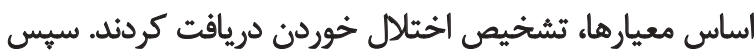

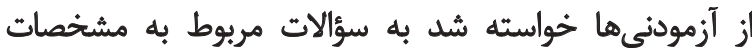

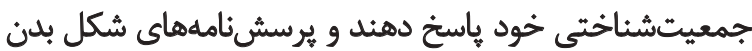

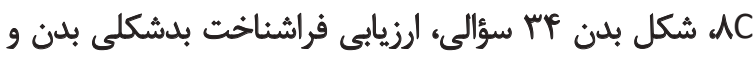

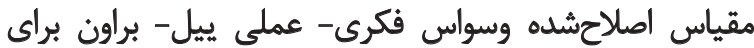
اختلال بد شكلى بدن رانيز تكميل كنيند.

\section{"يرسشنامه شكل بدن (BSQ-AC)AC}

هرسشنامه BSQ-AC نسخه كوتاه برسشنامه شكل بدن و

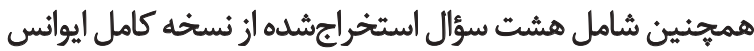

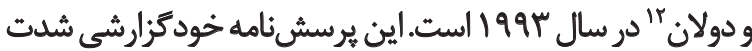

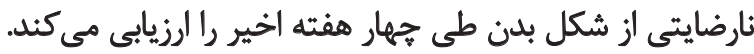

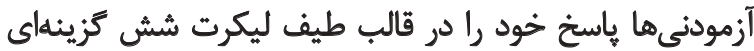

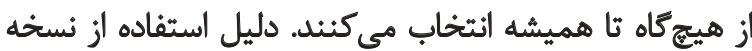
BSQ-AC

11. Body Shape Questionnaire-8C (BSQ-8C)

12. Evans and Dolan
طراحى كردند. هدف اين يرسشنامه اندازهكيرى نكرانى آزمودنى

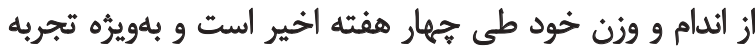

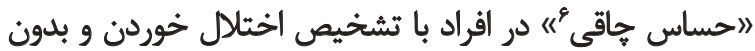

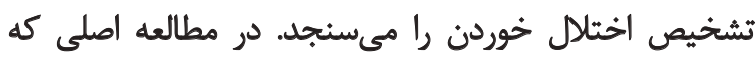

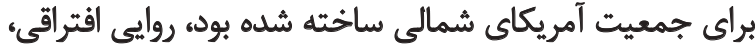

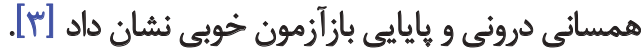
از مزيت اين يرسشنامه طرح سؤال هايى است كه سندرمهاى

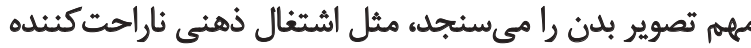

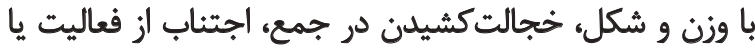

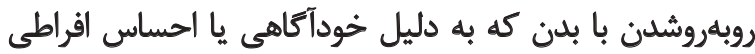

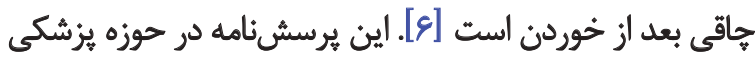

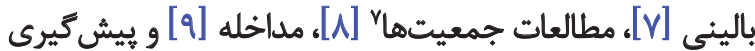

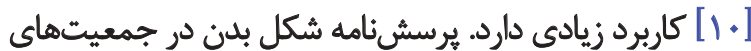

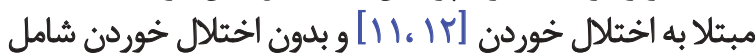

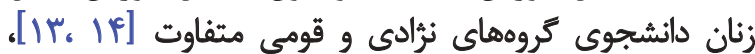

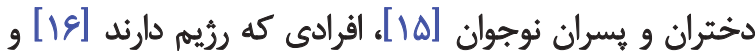
زنان سال خورده [IV] بسيار استفاده شده است.

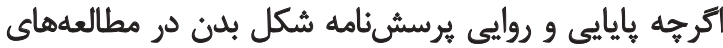

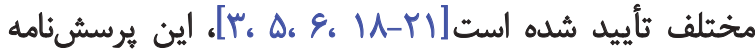

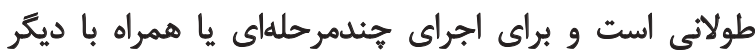

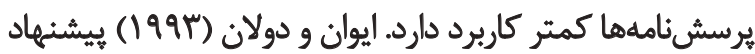

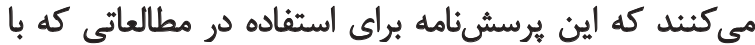

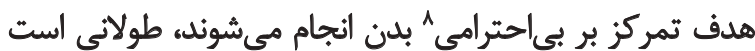

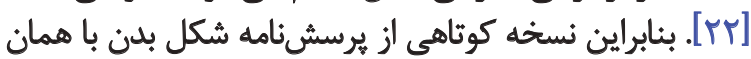

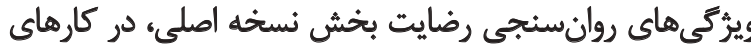

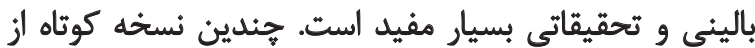

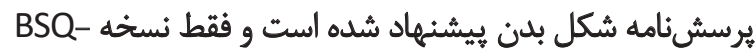

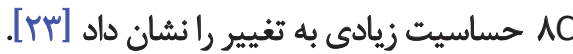

توجه به اين نكته اهميت دارد كه نارضايتى از شكل بدن

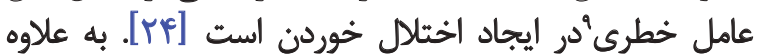

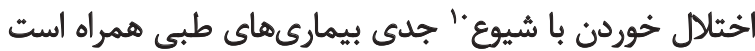

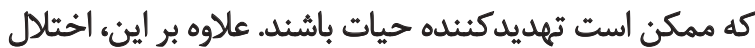

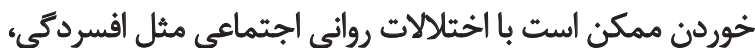

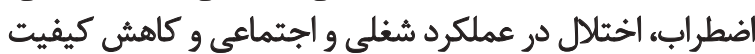

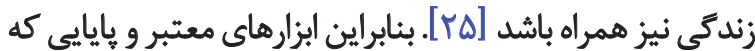

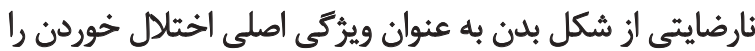

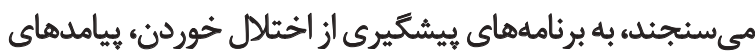

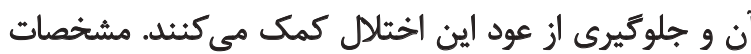

6. Feeling fat

7. Population studies

8. Disparagement

9. Risk factor

10. Morbidity 
آن با مقياس اصلاحشده وسواس فكرى- عملى ييل - براون براى

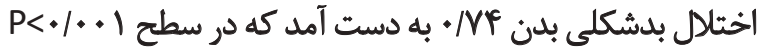

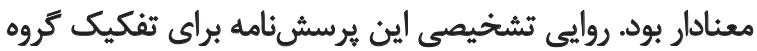

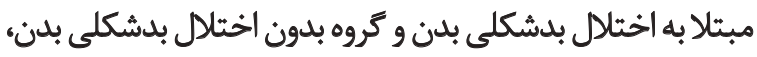

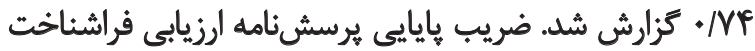

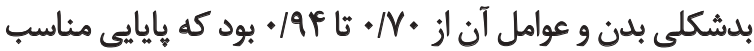

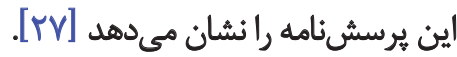

مقياس اصلاحشده وسواس فكرى-عملى ييل-براون براى اخثنال بدشكلى بلن (YBOCS-BDD)

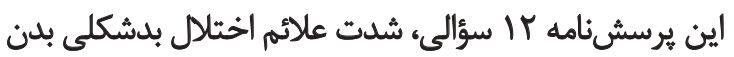

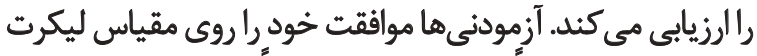

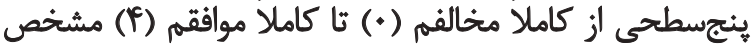

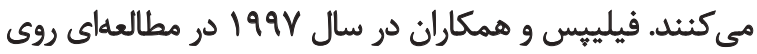

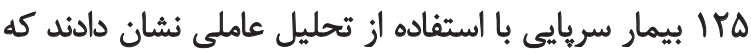

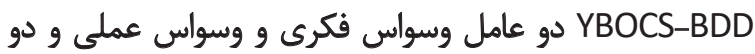

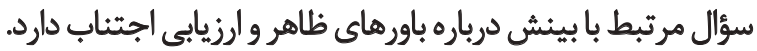

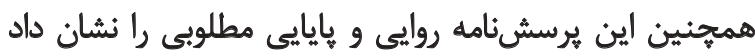

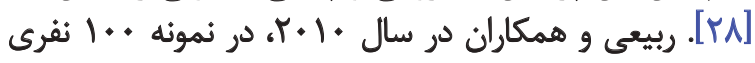

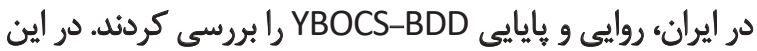

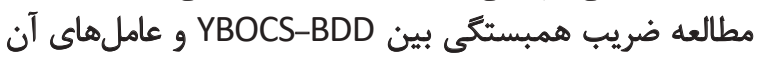

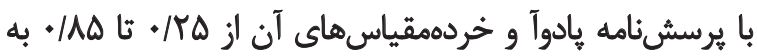

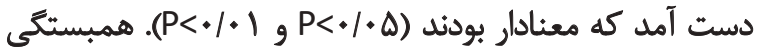

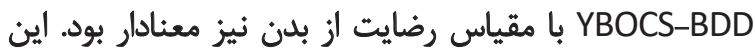

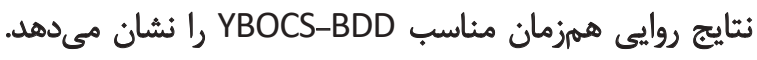

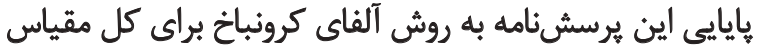

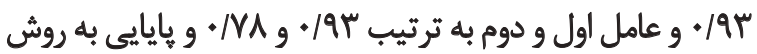

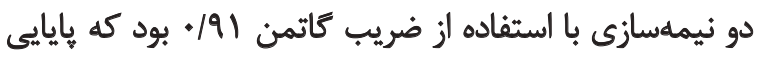

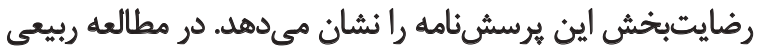

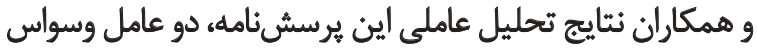

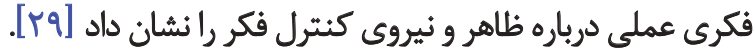

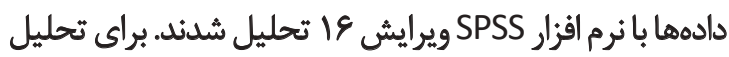

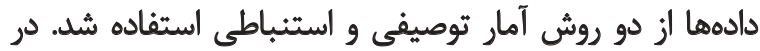

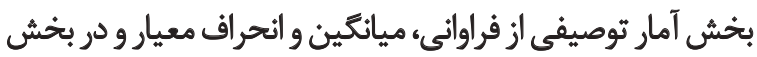

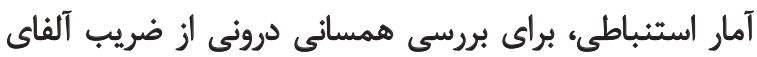

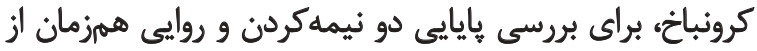

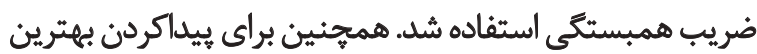

نقطهى برش اين يرسش نامه از منحنى راك استفاده شد.

Latiot

بر اساس مصاحبه تشخيصي دو روانشناس باليني به طور

19. Yale-Brown Obsessive Compulsive scale for Body Dysmorphic Disorder (YBOCS-BDD)

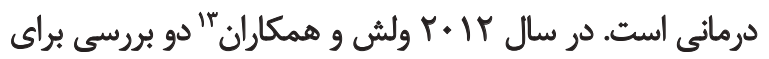

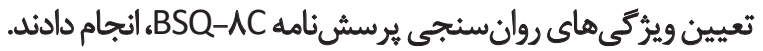

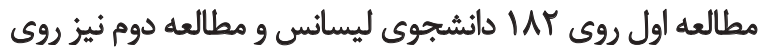

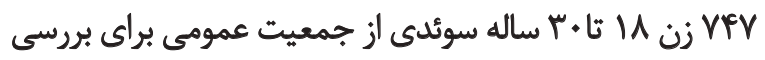

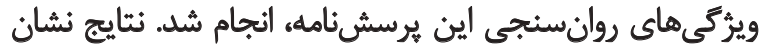

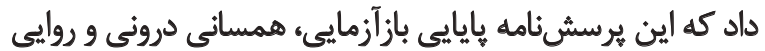

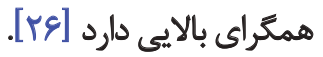

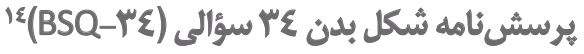

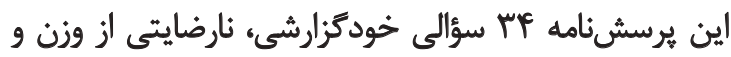

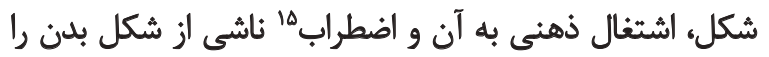

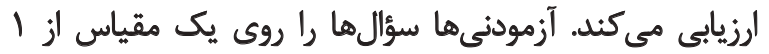

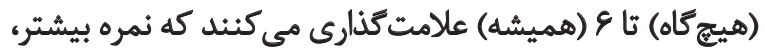

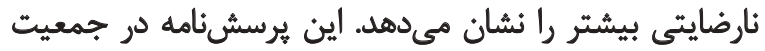

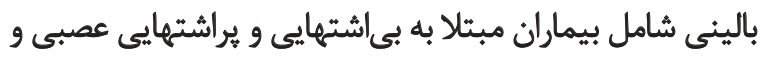

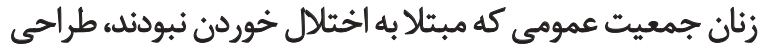

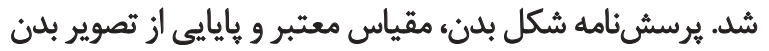

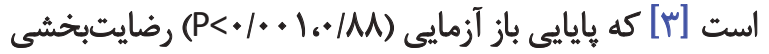

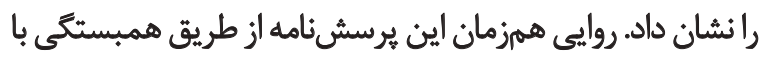

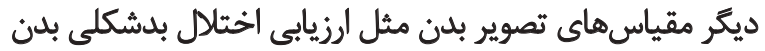

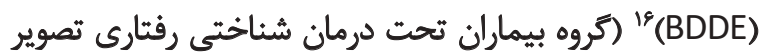

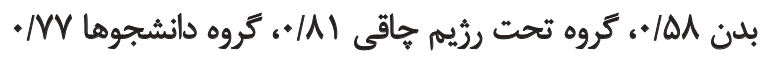
و كروه كارمندان دانشعاه

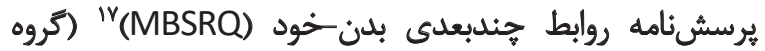

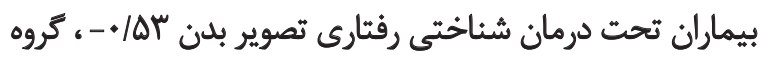

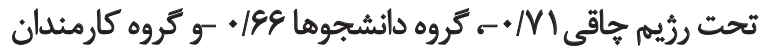

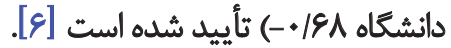

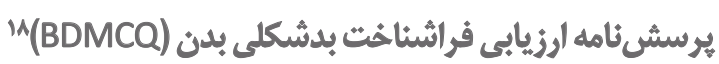
اين يرسش نامه آب سؤالى شامل جهار عامل است: راهبردهاى

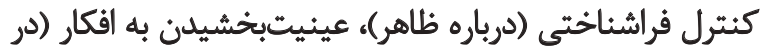

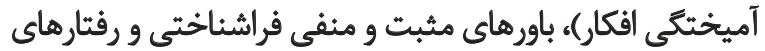

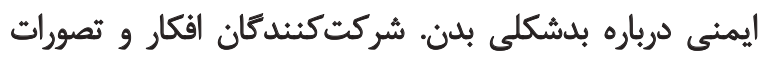

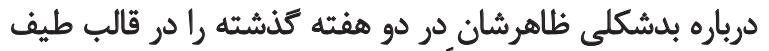

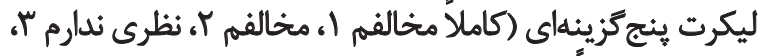

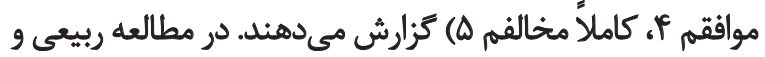

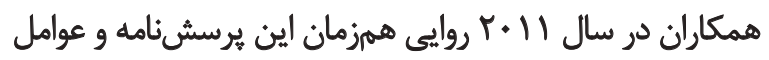

\section{Welch et al.}

14. Body Shape Questionnaire-34 (BSQ-34)

15. Distress

16. Body Dysmorphic Disorder Examination( BDDE)

17. Multi Dimensional Body-Self Relation Questionnaire (MBSRQ)

18. Body Dysmorphic Meta Cognition Questionnaire (BDMCQ) 
جدول ا. ميائكين و انحراف استاندارد سن، قد و وزن

ميانكين (انحراف استاندارد)

\begin{tabular}{|c|c|c|}
\hline افراد بدون تشخيص اختلال خُوردن & افراد با تشخيص اختلال خوردن & \\
\hline rq(NAA) & $r \cdot(A)(E / \Delta F)$ & سن ان \\
\hline $19 \cdot N A(8 / 19)$ & $\mid g T / T V(\& / g Y)$ & قد (سانتى متر) \\
\hline $\operatorname{gg/gr(1)/\Delta )}$ & $W / \&$ (q) & وزن (كيلوكرم) \\
\hline
\end{tabular}

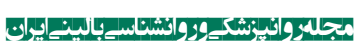

جدول Y. ضرايب بايايى يُرسشنامه شكل بدن AC

\begin{tabular}{|c|c|c|}
\hline ضريب دو نيمه سازى & ضريب آلفاى كرونباخ & \\
\hline$\cdot M^{f}$ &.$/ v 9$ & يرسش نامه شكل بلن AC \\
\hline
\end{tabular}

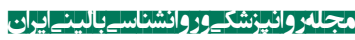

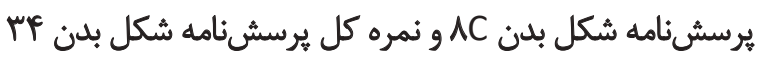

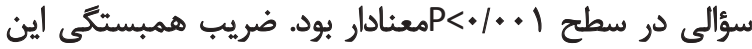

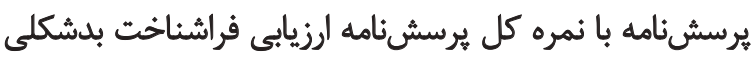

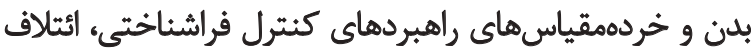

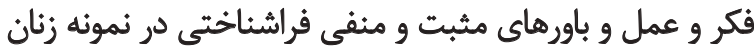

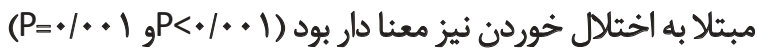

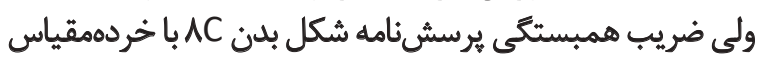

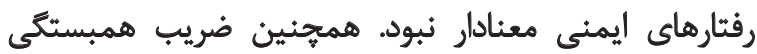

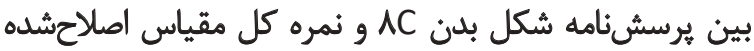

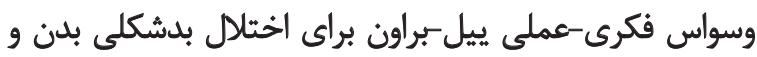

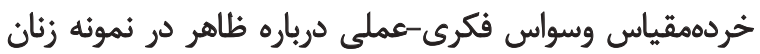

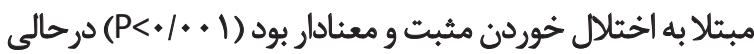

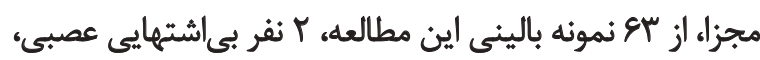

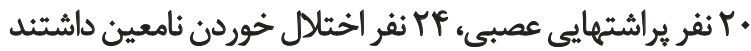

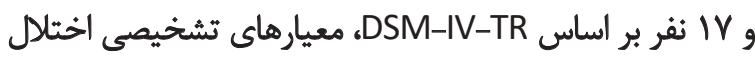

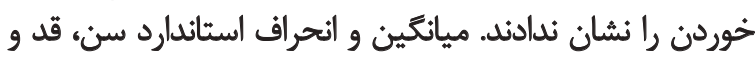
وزن نمونه تحت مطالعه در جدول شماره الرائه إئه شده است. براى بررسى روايى و پايايى برسش

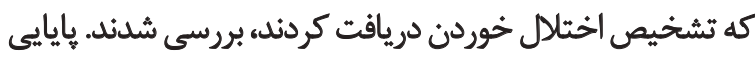

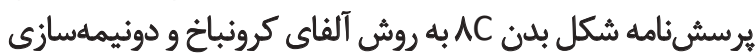

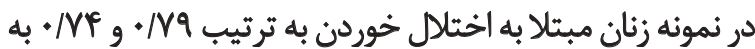

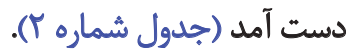
با توجه به يافتههاى جدول شماره با، ضريب همبستكى بين

جدول ". روايي همرزمان برسشنامه شكل بدن AC در نمونه زنان مبتّلا به اختلال خوردن

\begin{tabular}{|c|c|c|c|}
\hline سطع معنادارى & ضريب همبستكى & & مقياس \\
\hline$<+1 *+1$ & .191 & & يرسش ئامه شكل بلن به سؤالى \\
\hline $.1 . .1$ & .109 & كل - - - مل & \multirow{5}{*}{ برسش نامه ارزيابى فراشناخت بدشكلى بلن } \\
\hline $.1+.1$ & $\cdot / 0 Y$ & راهبردهاى كنترل فراشناختي & \\
\hline$+\infty 1$ &.$/ \Delta F$ & أتثلاف فكر و عمل & \\
\hline $.1 .+1$ &.$/ P V$ & باورهاي مثبت و هنفى فراشثاختي & \\
\hline .1 .09 &.$/ M A$ & رفتارهاى ايمنى & \\
\hline $.1 \cdot+1$ & $\cdot / 1 M$ & كل & \multirow{3}{*}{ 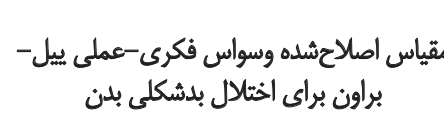 } \\
\hline$\%+1$ & .180 & وسواس فكرى - عملى درباره ظاهر & \\
\hline - /Pra &.$/ N T$ & ثيروى كتترل فكر & \\
\hline
\end{tabular}




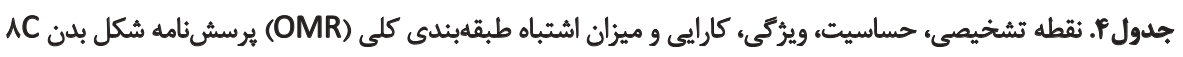

\begin{tabular}{|c|c|c|c|c|c|c|}
\hline ميزان اثشتباه طبقهبندى كلى & كاراين & ويزّكى & حساسيث & سطع زير منحنى & نقطه برش & \\
\hline.$/ r q$ & $\cdot M$ & $.18 V$ & - $1 \mathrm{~A}$ & . NE & $r E / \Delta$ & برئن بامه شكل \\
\hline
\end{tabular}

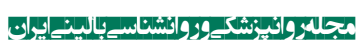

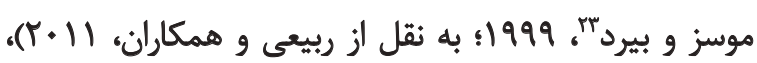

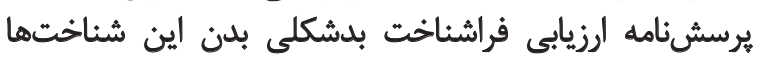

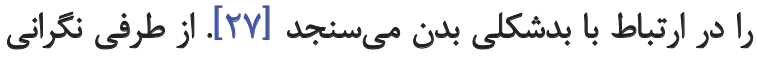

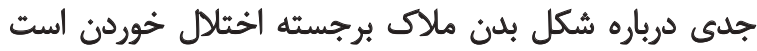

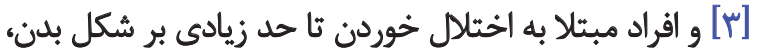

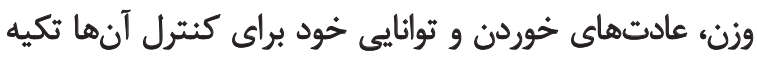

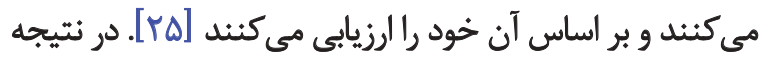

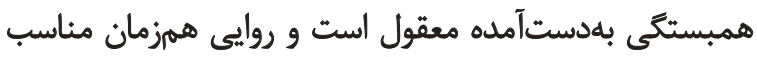

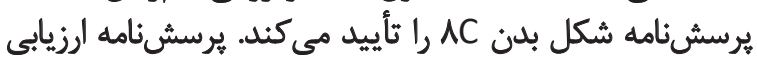

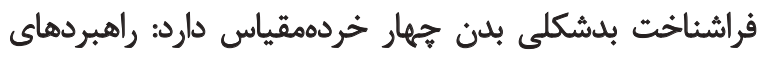

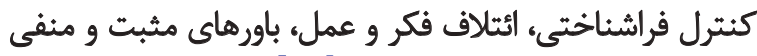

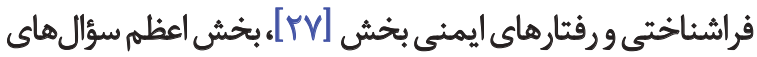

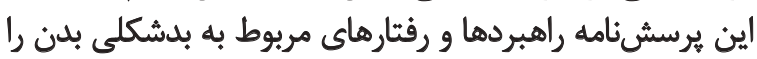

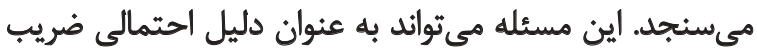

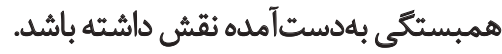

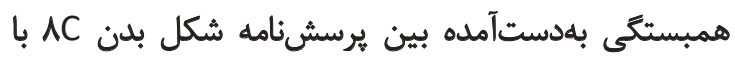
مقياس اصلاحشده وسواس فكرى-عملى ييل -براون براى اختيلال

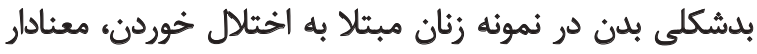

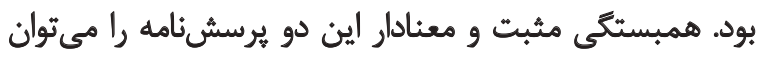

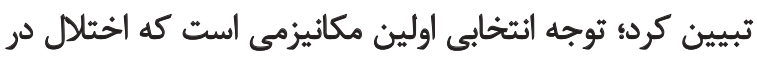

\section{Moses and Baird}

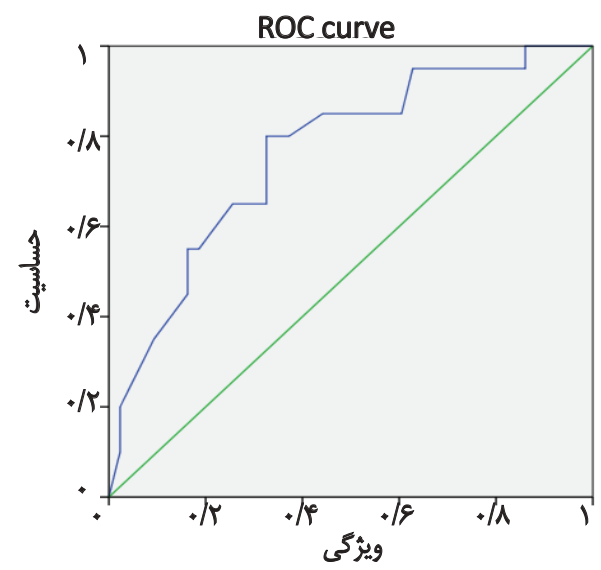

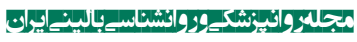

تصوير ا. نمودار راك يرسشنامه شكل بلن ئ Diagonal segments are produced by ties
كه ضريب همبستخى اين يرسشنامه با خرده مقياس نيروى كنترل فكر معنادار نبود.

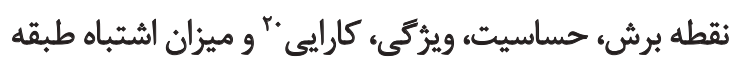

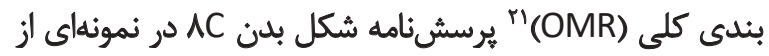
زنان مبتلا به اختلال خوردن در جدول شماره أ و تصوير شماره

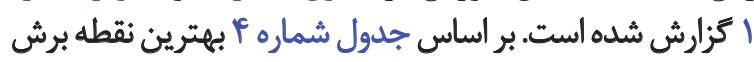

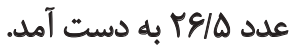

ث:

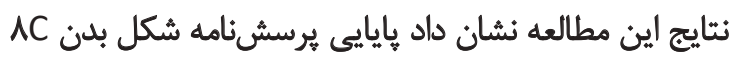

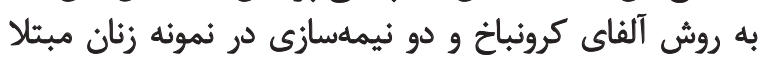

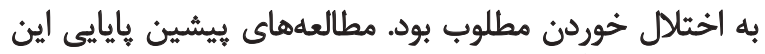

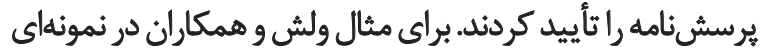

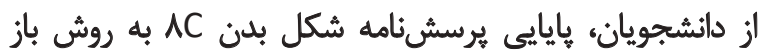

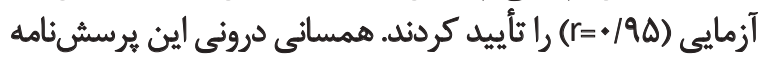
با روش آلفاى كرونباخ در نمونهاى از دانشجائ

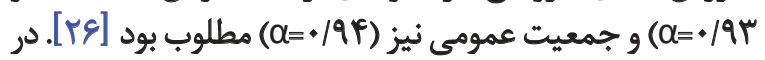

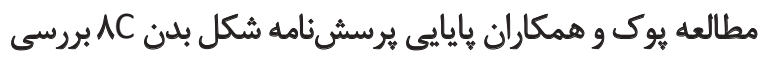

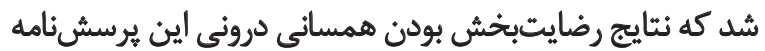

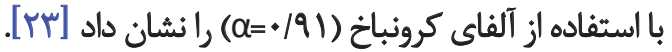

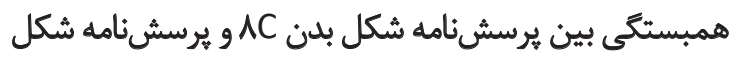

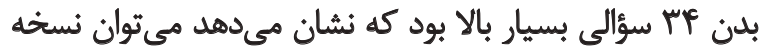

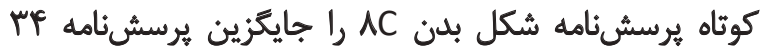

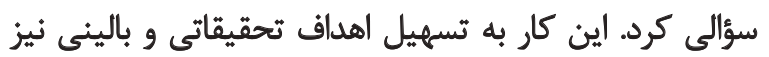

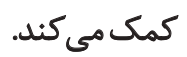

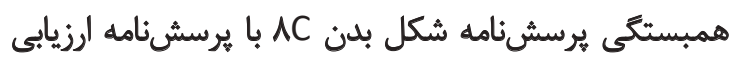

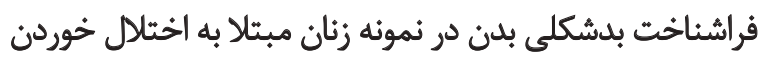

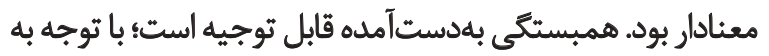

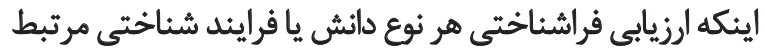

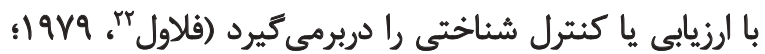

20. Efficacy

21. Overall Misclassification Rate (OMR)

22. Flavell 
بلن AC همبستكى بالاى اين يرسشنامه با نسخه اصلى و

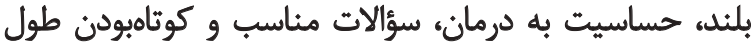

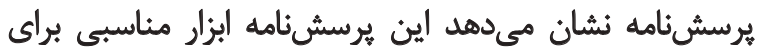

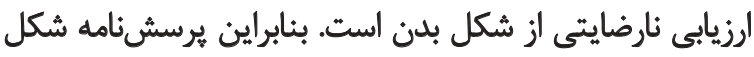

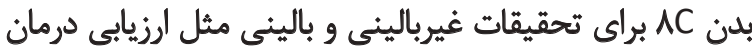

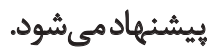

\section{نتيجليَيرى}

مطالعه حاضر در نمونهاي از زنان مبتلا به اختالال خوردن به به بـائ

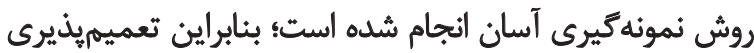

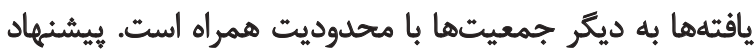

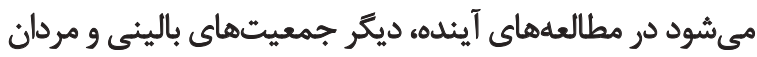

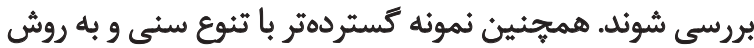

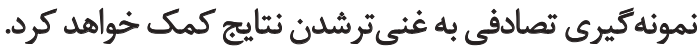

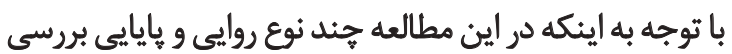

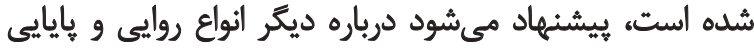
يرسشنامه شكل بدن AC مطالعاتى انجام شود.

$$
\text { سياسِّرًأرى }
$$

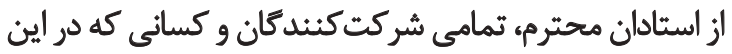

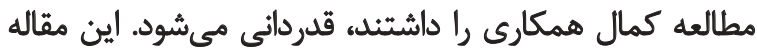

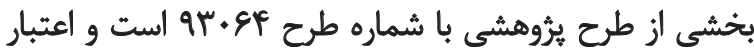

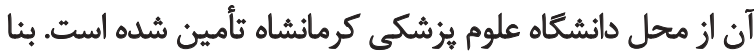

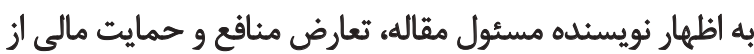
يرؤوهش وجود نداشته است.
تصوير بلن را حفظ مي كنده توجه انتخابي به بخش هايى از بلن،

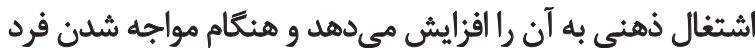

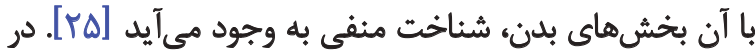

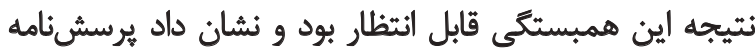

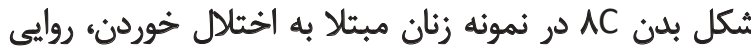

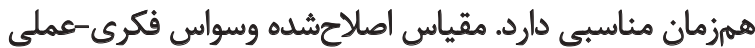

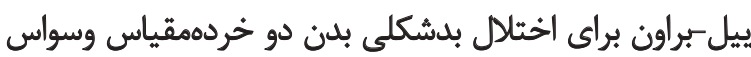

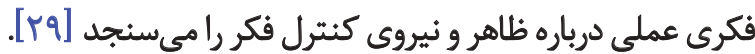

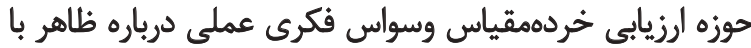

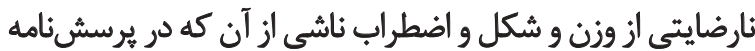

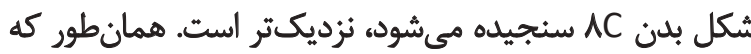

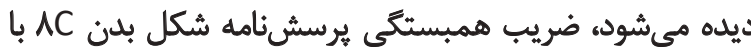

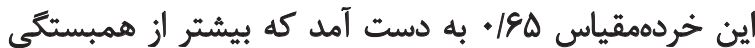

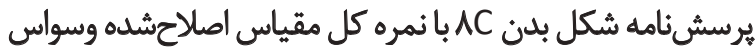

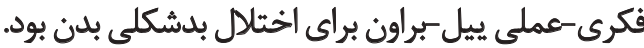

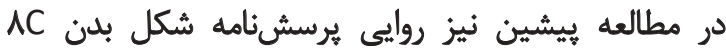

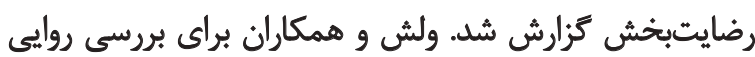

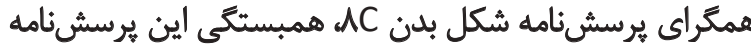

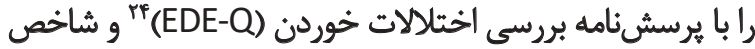

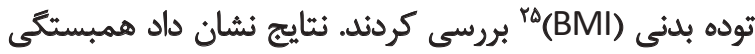

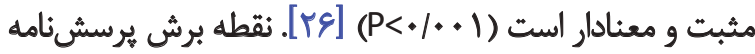

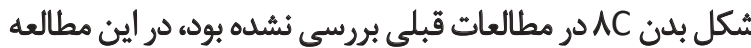

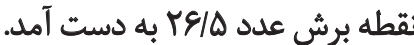

اين مطالعه كه با هدف كسترش اطلاعات مربوط به بدن و

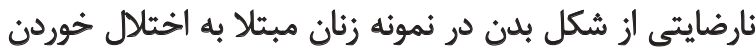

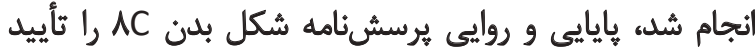

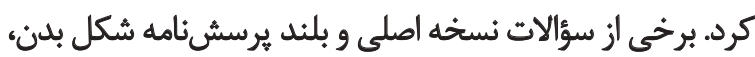
براى مردان كمتر مناسب بود، در نتيجه استفاده از ازئ اين نسخه

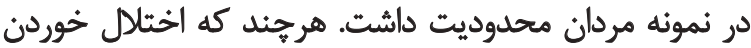

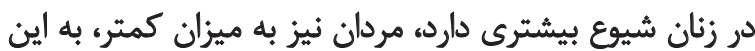

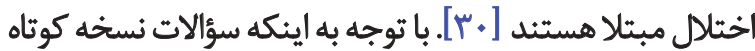

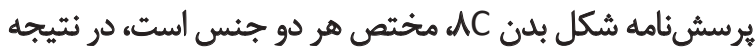

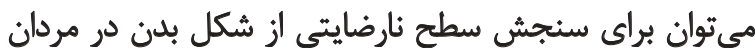

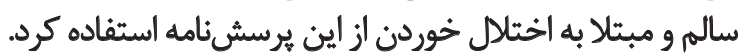

سؤالهاي يرسشنامه شكل بدن AC احساس فرد درباره

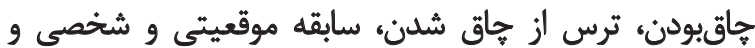

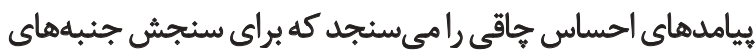

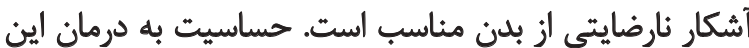

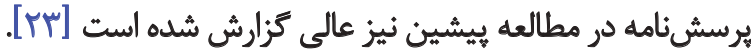

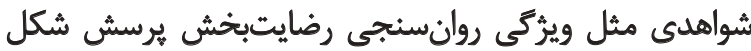

24. Eating Disorder Examination Questionnaire (EDE-Q) 25. Body Mass Index (BMI) 


\section{References}

[1] Slade PD. Body image in anorexia nervosa. The British Journal of Psychiatry. 1988; (2):20-2. PMID: 3072049

[2] Slade PD. What is body image? Behaviour Research and Therapy. 1994; 32(5):497-502. doi: 10.1016/00057967(94)90136-8

[3] Cooper PJ, Taylor MJ, Cooper Z, Fairbum CG. The development and validation of the body shape questionnaire. International Journal of Eating Disorders. 1987; 6(4):485-94. doi: 10.1002/1098-108x(198707)6:4<485::aideat2260060405>3.0.co;2-o

[4] Garner DM, Garfinkel PE. Body image in anorexia nervosa: Measurement, theory and clinical implications. The International Journal of Psychiatry in Medicine. 1982; 11(3):263-84. doi: 10.2190/r55q-2u6t-lam7-rqr7

[5] Ghaderi A, Scott B. The reliability and validity of the Swedish version of the Body Shape Questionnaire. Scandinavian Journal of Psychology. 2004; 45(4):319-24. doi: 10.1111/j.1467-9450.2004.00411.x

[6] Rosen JC, Jones A, Ramirez E, Waxman S. Body shape questionnaire: Studies of validity and reliability. International Journal of Eating Disorders. 1996; 20(3):3159. doi: 10.1002/(sici)1098-108x(199611)20:3<315::aideat11>3.3.co;2-n

[7] Hrabosky JI, Grilo CM. Body image and eating disordered behavior in a community sample of Black and Hispanic women. Eating Behaviors. 2007; 8(1):106-14. doi: 10.1016/j. eatbeh.2006.02.005

[8] Lundgren JD, Anderson DA, Thompson JK. Fear of negative appearance evaluation: Development and evaluation of a new construct for risk factor work in the field of eating disorders. Eating Behaviors. 2004; 5(1):75-84. doi: 10.1016/ s1471-0153(03)00055-2

[9] Franko DL, George JB. A pilot intervention to reduce eating disorder risk in Latina women. European Eating Disorders Review. 2008; 16(6):436-41. doi: 10.1002/erv.891

[10] Sepúlveda AR, Carrobles JA, Gandarillas A, Poveda J, Pastor V. Prevention program for disturbed eating and body dissatisfaction in a Spanish university population: A pilot study. Body Image. 2007; 4(3):317-28. doi: 10.1016/j. bodyim.2007.05.001

[11] Smith FT, Hardman RK, Richards PS, Fischer L. Intrinsic religiousness and spiritual well-being as predictors of treatment outcome among women with eating disorders. Eating Disorders. 2003; 11(1):15-26. doi: 10.1080/10640260390167456-2199

[12] Bunnell DW, Cooper PJ, Hertz S, Shenker IR. Body shape concerns among adolescents. International Journal of Eating Disorders. 1992; 11(1):7983. doi: 10.1002/1098-108x(199201)11:1<79::aideat2260110111>3.0.co;2-v

[13] Schooler D, Monique Ward L, Merriwether A, Caruthers A. Who's that girl: television's role in the body image development of young white and black women. Psychology of Women Quarterly. 2004; 28(1):38-47. doi: 10.1111/j.14716402.2004.00121.x
[14] Warren CS, Cepeda Benito A, Gleaves DH, Moreno S, Rodriguez S, Fernandez MC, et al. English and Spanish versions of the Body Shape Questionnaire: Measurement equivalence across ethnicity and clinical status. International Journal of Eating Disorders. 2008; 41(3):265-72. doi: 10.1002/eat.20492

[15] Caradas AA, Lambert EV, Charlton KE. An ethnic comparison of eating attitudes and associated body image concerns in adolescent South African schoolgirls. Journal of Human Nutrition and Dietetics. 2001; 14(2):111-20. doi: 10.1046/j.1365-277x.2001.00280.x

[16] Green MW, Rogers PJ. Impairments in working memory associated with spontaneous dieting behaviour. Psychological Medicine. 1998; 28(5):1063-70. doi: 10.1017/ s0033291798007016

[17] Hetherington MM, Burnett L. Ageing and the pursuit of slimness: dietary restraint and weight satisfaction in elderly women. British Journal of Clinical Psychology. 1994; 33(3):391-400. doi: 10.1111/j.2044-8260.1994.tb01135.x

[18] Di Pietro M, Silveira DXd. Internal validity, dimensionality and performance of the Body Shape Questionnaire in a group of Brazilian college students. Revista Brasileira de Psiquiatria. 2009; 31(1):21-4. doi: 10.1590/ s1516-44462008005000017

[19] Conti MA, Cordás TA, Latorre M do RD de O. A study of the validity and reliability of the Brazilian version of the Body Shape Questionnaire (BSQ) among adolescents. Revista Brasileira de Saúde Materno Infantil. 2009; 9(3):331-8. doi: 10.1590/s1519-38292009000300012

[20] Akdemir A, Inandi T, Akbas D, Karaoglan Kahilogullari A, Eren M, Canpolat BI. Validity and reliability of a Turkish version of the Body Shape Questionnaire among female high school students: Preliminary examination. European Eating Disorders Review. 2012; 20(1):e114-e5. doi: 10.1002/ erv.1106

[21] Lentillon-Kaestner V, Berchtold A, Rousseau A, Ferrand C. Validity and reliability of the French versions of the Body Shape Questionnaire. Journal of Personality Assessment. 2013; 96(4):471-7. doi: 10.1080/00223891.2013.843537

[22] Evans C, Dolan B. Body shape questionnaire: Derivation of shortened "alternate forms". International Journal of Eating Disorders. 1993; 13(3):31521. doi: 10.1002/1098-108x(199304)13:3<315::aideat2260130310>3.0.co;2-3

[23] Pook M, Tuschen-Caffier B, Brähler E. Evaluation and comparison of different versions of the Body Shape Questionnaire. Psychiatry Research. 2008; 158(1):67-73. doi: 10.1016/j.psychres.2006.08.002

[24] Oliveira FPD, Bosi MLM, Vigário PdS, Vieira RdS. [Eating behavior and body image in athletes (Portuguese)]. Revista Brasileira de Medicina do Esporte. 2003; 9(6):348-56. doi: $10.1590 /$ s1517-86922003000600002

[25] Cash TF, Smolak L. Body image, second edition: A handbook of science, practice, and prevention. New York: Guilford Publications; 2011.

[26] Welch E, Lagerström M, Ghaderi A. Body Shape Questionnaire: Psychometric properties of the short version (BSQ-8C) and norms from the general Swedish popula- 
tion. Body Image. 2012; 9(4):547-50. doi: 10.1016/j.bodyim.2012.04.009

[27] Rabiei M, Salahian A, Bahrami F, Palahang H. [Construction and standardization of the body dysmorphic metacognition questionnaire (Persian)]. Journal of Mazandaran University of Medical Sciences. 2011; 21(83):43-52.

[28] Phillips KA, Hollander E, Rasmussen SA, Aronowitz BR, DeCaria C, Goodman WK. A severity rating scale for body dysmorphic disorder: development, reliability, and validity of a modified version of the Yale-Brown Obsessive Compulsive Scale. Psychopharmacology Bulletin. 1997; 33(1):17-22. PMID: 9133747

[29] Rabiee M, Khorramdel K, Kalantari M, Molavi H. [Factor structure, validity and reliability of the modified Yalebrown obsessive compulsive scale for body dysmorphic disorder in students (Persian)]. Iranian Journal of Psychiatry and Clinical Psychology. 2010; 15(4):343-50.

[30] Sadock BJ, Sadock VA. Kaplan \& Sadock's synopsis of psychiatry: Behavioral sciences/clinical psychiatry. Philadelphia: Lippincott Williams \& Wilkins; 2007. 
